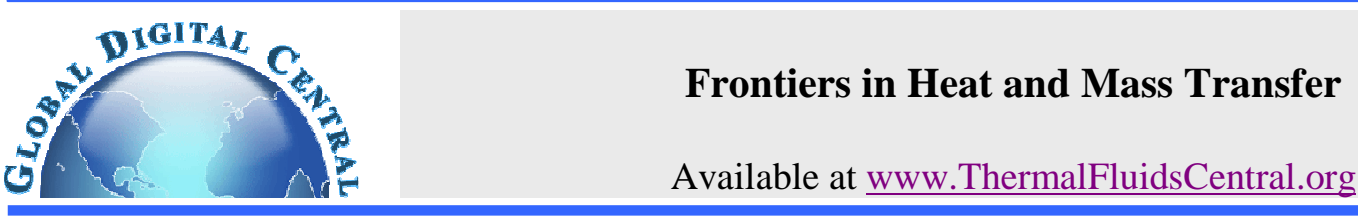

\title{
CHARACTERISTICS OF MICROLAYER FORMATION AND HEAT TRANSFER IN MINI/MICROCHANNEL BOILING SYSTEMS: A REVIEW
}

\author{
Yaohua Zhang $^{\mathrm{a},}$ * Yoshio Utaka $^{\mathrm{b}}$ \\ ${ }^{a}$ Graduate School of Engineering, Yokohama National University, Tokiwadai, Hodogaya, Yokohama 240-8501, Japan \\ ${ }^{\mathrm{b}}$ Faculty of Engineering, Yokohama National University, Tokiwadai, Hodogaya, Yokohama 240-8501, Japan
}

\begin{abstract}
This paper reviews recent research on microlayer formed by confined vapor bubbles during boiling in mini/microchannels. Experimental measurements, simulations and theoretical studies are described and compared. As a reference to clarify the mechanism for the formation of a microlayer, Taylor flow (i.e. elongated bubble flow in mini/micro circular tubes under adiabatic conditions and at $R e<1$ ) and elongated bubble flow at high velocity, with consideration of the influence of inertia, are also reviewed. Compared to the steady adiabatic conditions, one of the distinct points for the boiling condition is that the bubble grows exponentially due to rapid evaporation of the microlayer. A pattern of two regions (i.e., surface tension-viscosity controlled and boundary layer controlled regions) seems to be presently acceptable to describe microlayer formation for a wide range of bubble growth velocity. In addition, the effect of microlayer evaporation on the boiling heat transfer in mini/microchannel is reviewed.

Keywords: Microlayer, boiling, mini/Microchannel, acceleration
\end{abstract}

\section{INTRODUCTION}

In recent years, the phenomena of boiling in microchannels has attracted the interest of numerous researchers, due to the rapid development of micro/mini devices used in several engineering applications, including compact, high-heat-flux heat exchangers and cooling systems for various devices such as high-performance microelectronic devices, microelectromechanical systems, and aerospace components. Surface tension affects the lateral and axial distribution of liquid and vapor in mini/microchannels, so that the physics of boiling differs considerably from that in macrochannels. $\mathrm{Qu}$ and Mudawar (2003) reported that, in contrast to conventional channels, local heat transfer coefficients in microchannels have been shown experimentally to be almost exclusively dependent on the heat flux and saturation pressure, and instead decrease with the vapor quality.

Therefore, the existing empirical correlations and models for heat transfer developed for macrochannels are not reliable guides for mini/microchannels, because they are based on conventional nucleate boiling and convective heat transfer models. Instead, Jacobi and Thome (2002) and Thome et al. (2004) proposed that transient evaporation of thin liquid films trapped between the bubble and heating surface is the dominant heat transfer mechanism in the mini/microchannel heat transfer model. Parametric studies have shown that the Jacobi-Thome model (2002) predicted several independent experimental results quite well with the assumption of nucleation superheat and an initial thin liquid film thickness. Thus, it is very important to clarify the behavior of the microlayer and to develop a method to predict its thickness.

Kandlikar (2002), Cheng et al. (2007) and Ong and Thome (2011) have presented summaries on research of evaporation in microchannels. These reviews addressed channel classifications based on different methods, of which the typical methods are shown in Table 1. The confinement number, $C o$ and Bond number, Bo are respectively defined as

$$
\begin{gathered}
C o=\frac{1}{D_{h}} \sqrt{\frac{\sigma}{g\left(\rho_{L}-\rho_{V}\right)}} \\
B o=\frac{g\left(\rho_{L}-\rho_{V}\right) D_{h}{ }^{2}}{\sigma}
\end{gathered}
$$

where $D_{h}$ is the hydraulic diameter of the channel; $g$ is gravitational acceleration; $\sigma$ is surface tension; and $\rho_{L}$ and $\rho_{V}$ are the densities of the liquid and the vapor, respectively. There have been a number of attempts to define a criterion for channel scale, ranging from that based on the physical hydraulic diameter to a general definition.

Wayner (1999) and Stephan (2002) gave comprehensive reviews of articles regarding microscale evaporation with consideration of the intermolecular forces. Under this condition, an augmented YoungLaplace model is employed by introducing a disjoining pressure, such that, $P_{V}-P_{L}=P_{c}+P_{d}$ where is the capillary pressure (given by the product of interfacial curvature $K$ and surface tension coefficient $\sigma$ ); $P_{d}=A / \delta^{3}$ is the disjoining pressure and (where $A$ is the dispersion constant and $\delta$ is the film thickness); $P_{V}$ and $P_{L}$ are the vapor and liquid pressures, respectively. The evaporating meniscus in a microchannel can then be generally divided into three regions, as illustrated in Fig. 1: an adsorbed or non-evaporating region with a nanometer order thickness of $\delta$ in which liquid is adsorbed on the wall; a transition region in which the effects of long-range molecular forces (disjoining pressure) are felt; and an intrinsic meniscus region, where capillary forces dominate. With respect to the boiling in mini/microchannels (in

\footnotetext{
*Corresponding author. Email: yhzhang1981ch@yahoo.co.jp
} 


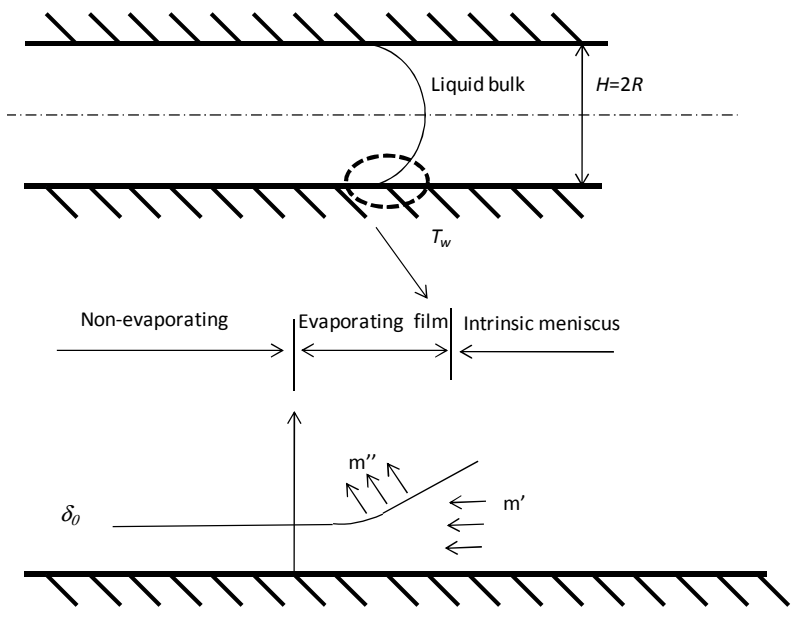

Fig. 1 Schematic diagram for an evaporating meniscus in a channel by Stephan (2002).

several hundred micrometers of channel), the microlayer formed between the confined bubble and heating surface is of micrometer order and becomes thin due to evaporation. Generally, most of the boiling heat transfer process is considered to be completed before the microlayer decreases to a nanometer order liquid layer, during which the effect of disjoining pressure can be neglected.

The present study focuses on the initial microlayer (with micrometer order thickness), which is defined as the thin liquid layer formed just behind the moving bubble tip, and where the surface tension effect becomes dominant. The aim of this paper is to present typical research related to the microlayer formed in mini/microchannels during boiling or under adiabatic conditions (which can assist understanding the formation mechanism and characteristics of microlayers under boiling), and to propose areas available for further investigation.

\section{MEASUREMENT OF MICROLAYER THICKNESS}

Despite the importance of predicting the heat transfer characteristics, relatively few studies have investigated the microlayer thickness in microscale boiling systems. This is partially because boiling in microscale systems is a developing area of research. The main reason is that the spatial and temporal scales involved make it inherently difficult to develop high-response, non-contact measurement methods. In microscale boiling systems, thermal transport occurs over considerably longer time scales than the fluidic response, as reported by Miler et al. (2010). It is thought that the microlayer thickness immediately after the passage of the bubble tip is determined by the kinetic interface behavior during bubble growth and that it is not affected by evaporation. Therefore, investigation of the microlayer thickness under adiabatic conditions (even studies that do not explicitly address boiling in mini/microchannels) may provide valuable insight.

Taylor (1961), a pioneer in this research, measured the amount of liquid remaining on a tube wall when air is blown into a glass tube (diameter: $1.5-3 \mathrm{~mm}$, length: $105 \mathrm{~mm}$ ) that had been previously filled with a glycerin-water solution. He did this by determining the mass difference between the inlet and outlet over a specific time. Although this experiment give the residual liquid fraction on the tube wall rather than the liquid layer thickness, it is possible to calculate the former from the latter. Extending Taylor's experiment, Aussillous and Quere (2000) blew air into transparent polymeric tubes of $0.42,0.62,0.78$, and $1.46 \mathrm{~mm}$ in diameter containing drops (with initial lengths of 50$100 \mathrm{~mm}$ ). The positions of the front and rear meniscuses of the drops were measured as a function of time using a camera. The deposition velocity was deduced from the recorded videos and the film thickness was calculated from the rate at which the drop length decreased. Results were obtained for high velocities by using a low- viscosity liquid, which extended Taylor's experiment to the low deposition rate limit and made it possible to stress the influence of inertia on the film thickness.

Katto and Shoji (1970) utilized radial spreading of a flattened air bubble that was artificially produced by injecting air into a narrow space between a pair of parallel disks (diameter: $30 \mathrm{~mm}$, gap size: 0.5 $\mathrm{mm}$ ) filled with a transparent liquid (water or methanol), and the behavior of the microlayer thus created on a solid surface was observed. Air bubble growth and the microlayer thickness were simultaneously measured using a light-interference technique and high-speed photography. They found that the increasing rate of the microlayer thickness with the radial distance from the center becomes greater as the spreading velocity of the bubble increases. Their experiments were performed for local spreading velocities within the range $0.05-0.75 \mathrm{~m} / \mathrm{s}$, which is considered to be a similar order as the bubbles growth rate in normal nucleate boiling, but slower than that in a microscale boiling system.

Tibirica et al. (2010) presented a comprehensive literature review of the methods that have been used to measure liquid film thickness from macro to microscale systems under various experimental conditions (i.e., not just internal two-phase flow, but also conditions such as spray cooling on a plate and film flow). In order to group these methods based on similar principles, the authors proposed the following classification: (1) acoustic methods based on ultrasonic waves (e.g. Lu et al., 1993; Carvalho et al., 2008); (2) electrical methods that apply an electrical potential difference between electrodes and measure the resulting current (e.g. Thwaites et al., 1976; Kang and Kim, 1992; Thorncroft and Klausner, 1997; Seleghim and Hervieu,1998; Kumar et al., 2002; Jong and Gabriel, 2003; Liu and Chen, 2007; Ameur et al., 2007); (3) optical methods based on light intensity attenuation, interface detection, total internal light reflection and laser focus displacement (e.g. Roy et al., 1986; Yu et al., 1996; Tasaki and Utaka, 2003; Ursenbacher et al., 2004; Shedd and Newell, 2004). Group (3) methods are preferable for microscale measurements, because they have almost no effect on the flow and fluid properties (provided the light intensity is sufficiently low that it does not alter the properties of the fluid).

In addition to the these studies, most of which were measured under steady adiabatic conditions, Moriyama and Inoue (1996) measured the thickness of a microlayer formed on a wall when a flattened bubble grows in a superheated liquid in the narrow gaps between two parallel glass plates. Experiments were performed using R113 as a test fluid for gap sizes of $0.1,0.2$, and $0.4 \mathrm{~mm}$. The liquid microlayer thickness was estimated from the transient temperature variation of the wall surface, by assuming that the heat removed from the wall is consumed by evaporation of the microlayer. They found that the interface traveling velocity increases monotonically with initial superheat, as shown in Fig (a), while Fig. 2(b) shows that the microlayer thickness has a maximum variation with initial superheat.

Utaka et al. (2006) measured the microlayer thickness formed between the heating surface and a confined vapor bubble during the microlayer growth process using the experimental apparatus shown in Fig. 3. Measurements were performed for water with the laser extinction method for gap sizes of $0.5,0.3$, and $0.15 \mathrm{~mm}$. Infrared laser with a wavelength of $3.39 \mu \mathrm{m}$ was launched through the microchannel measurement section and the attenuated signal was measured with an infrared detector. Lambert's law was employed to determine the microlayer thickness based on the transmittance ratio of the laser. They divided the variation of the microlayer thickness relative to the rate of bubble growth into two regions from the small velocity side; the region where the thickness increases linearly with increasing bubble forefront velocity and the region where the thickness is almost constant. The atter region appears at a bubble forefront velocity of approximately 


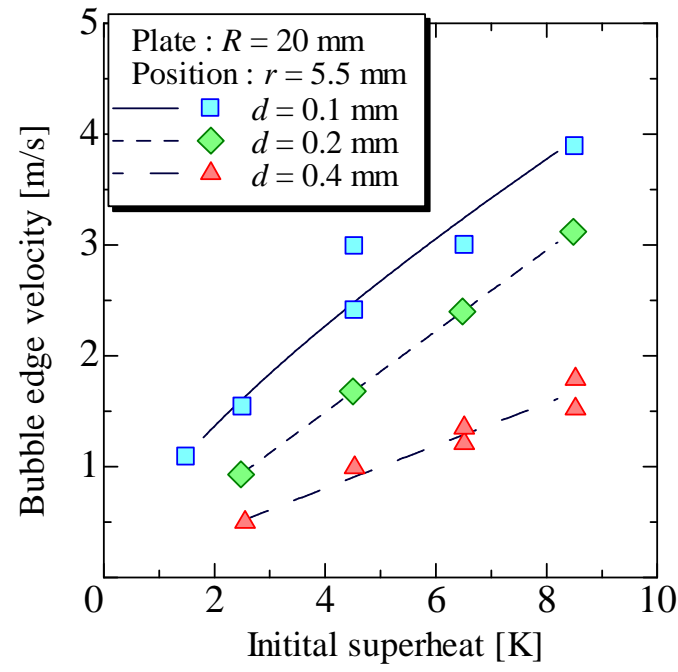

(a) Interface traveling velocity versus initial superheat

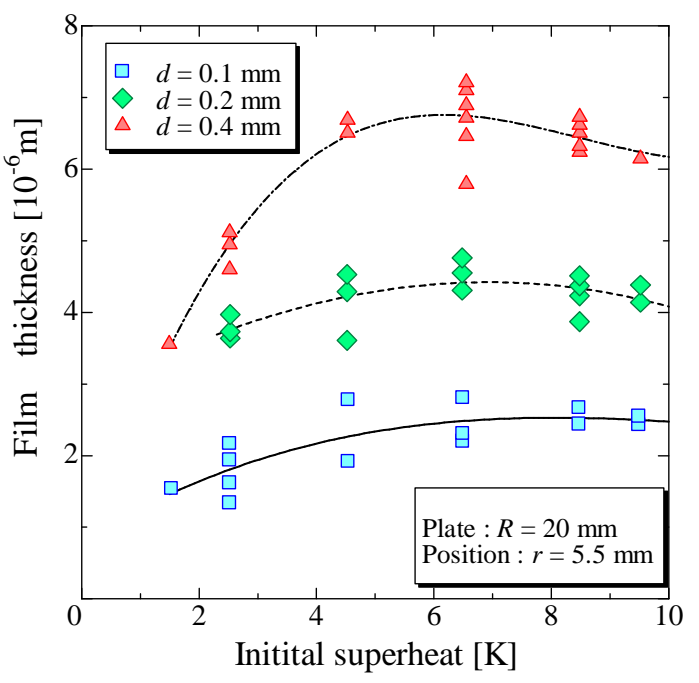

(b) Initial thickness of the microlayer versus initial superheat

Fig. 2 Experiment results from Moriyama and Inoue (1996).

$2 \mathrm{~m} / \mathrm{s}$ (see Fig. 4). Zhang et al. (2010a, 2010b) extended the study and measured the microlayer thickness of ethanol, toluene and HFE7200 by the same way and found that the traveling distance of the bubble interface has an apparent effect on the thickness of microlayer in the high speed region (see Fig. 5).

Using the laser focus displacement meter shown in Fig. 6, Han and Shikazono (2009) measured the thickness of microlayer formed slug flow under steady adiabatic conditions in Pyres glass tubes with diameters of $0.3,0.5,0.7,1.0$ and $1.3 \mathrm{~mm}$. Ethanol, water and FC-40 were employed as testing fluids. An actuator motor was used to control the velocity of the liquid from 0 to $7 \mathrm{~m} / \mathrm{s}$. The position of the target surface can be determined by the displacement of an objective lens moved by the tuning fork. The intensity of the reflected light becomes highest in the light-receiving element when the focus is obtained on the target surface. The objective lens is vibrated continually in the range of $\pm 0.3 \mathrm{~mm}$. The microlayer thickness is obtained from the measured values. In order to investigate the effect of acceleration on the formation of the microlayer, Han and Shikazono (2010a) measured the thickness of microlayers formed by air bubble motion with constant acceleration that is generated by a quick pull of the liquid using an actuator motor. They concluded that the increase of the microlayer with the bubble front velocity is restricted by the acceleration, as Fig. 7

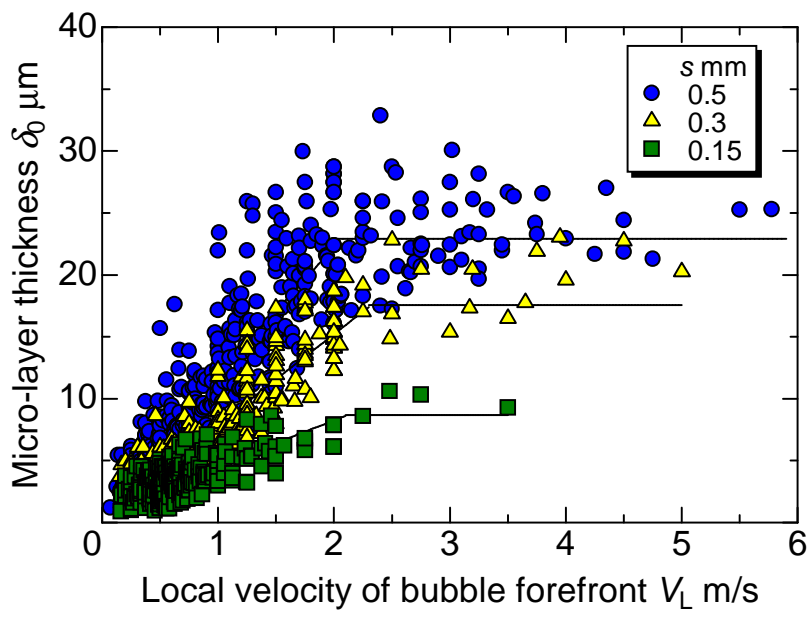

Fig.3 Experimental apparatus for measurement of the microlayer thickness in mini gaps by Utaka et al., (2006).

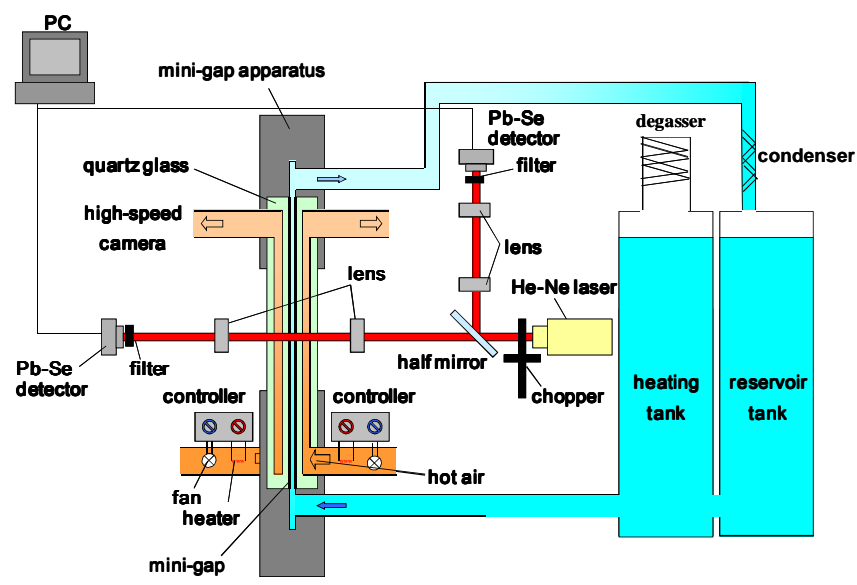

Fig. 4 Microlayer thickness and local velocity of bubble forefront for $s=0.15,0.3$ and $0.5 \mathrm{~mm}$ by Utaka et al. (2006).

shows for ethanol in a $D=0.7 \mathrm{~mm}$ tube, where $L$ is the bubble traveling distance.

From these results it can be concluded that the microlayer thickness is dependent on the position along the flow direction in the microchannel boiling system due to the effect of acceleration, which differs from the uniform thickness assumption under steady bubble motion. Therefore, reconsideration is required when the bubble travels with being accelerated, as shown (later) for boiling in mini/microchannels. Furthermore, measurement uncertainties still remain if comparing those measurement results. Because of the limited data number about the microlayer thickness measurements during boiling in microchannel, there are only a few cases to compare the independent data with various methods. Figure 8 shows experimental data for the initial microlayer thickness from Moriyama and Inoue (1996), Zhang and Utaka (2010a) for HFE7200 as a test fluid and Han and Shikazono (2010a) for FC40 (both of which have similar properties to R113, as shown in Table 2). Although the experimental conditions are not exactly the same, the microlayer thicknesses measured by Moriyama and Inoue (1996) are significantly (several times) thinner than those reported by Zhang and Utaka (2010a), and Han and Shikazono (2010a). Furthermore, the measurements of water with channel size of $0.5 \mathrm{~mm}$ by Zhang and Utaka (2010a) for parallel plate channel, and Han and Shikazono (2010a) for circular tube are plotted in Fig.9. 


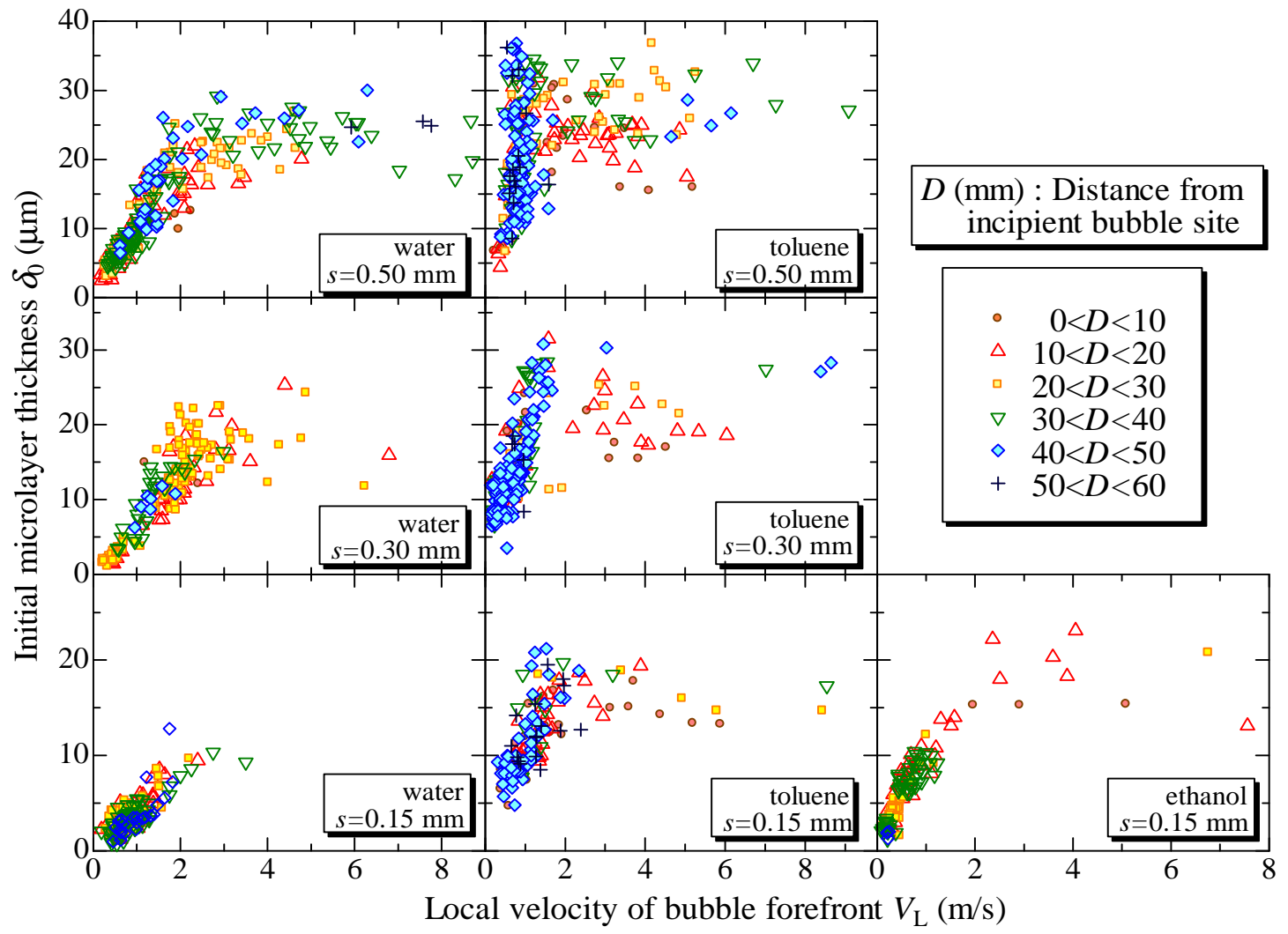

Fig. 5 Effect of bubble forefront traveling distance on the microlayer thickness by Zhang et al. (2010b).

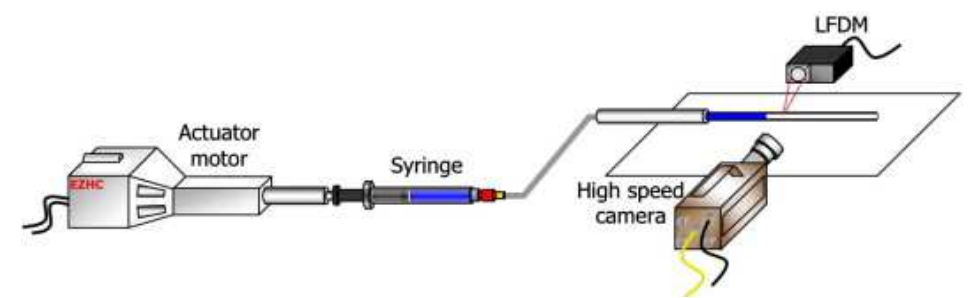

(a) Experimental apparatus

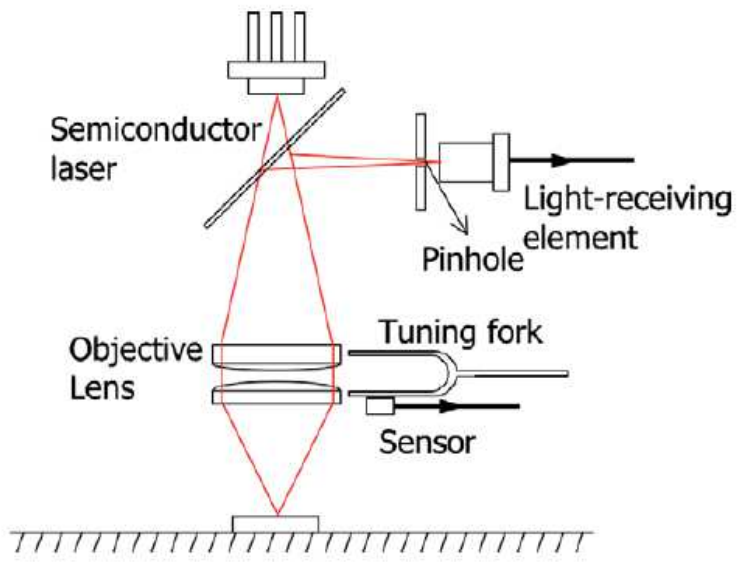

(b) Principle of the laser focus displacement meter

Fig. 6 Experimental apparatus for measurement of the microlayer thickness in microtubes by Han and Shikazono (2009).

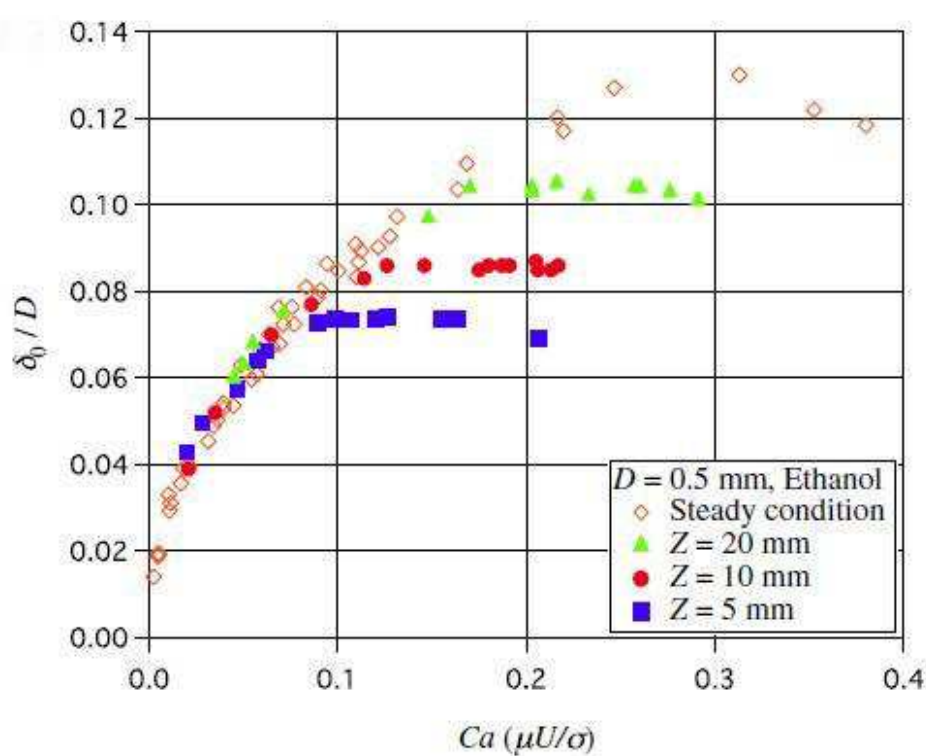

Fig. 7 Experimental results for ethanol under accelerated motion of an air bubble by Han and Shikazono (2010a).

Consistence can be found in the region of low velocity, while perhaps due to the different channel geometry and experimental condition (for example, the acceleration of vapor forefront) the data at region of high velocity disaccord a little with each other. 


\section{CHARACTERISTICS OF FORMATION AND CONFIGURATION OF A MICROLAYER}

The formation of microlayers in mini/microchannels could be considered to be the result of a balance between viscous, capillary and inertial forces, while their magnitudes are different depending on the bubble motion. For steady slow motion, i.e. the inertia could be assumed to be of minor importance, the system behavior would then governed by a balance between viscous and capillary forces. At faster steady flow, the effect of inertial force becomes non-negligible and steady, so that the Navier-Stokes equations must be employed instead of the Stokes equation for the former condition. As to the boiling condition, which is also the most unclear factor, the bubble undergoes significant volume change and the flow is highly transient as opposed to the steady or quasi-steady situations considered before.

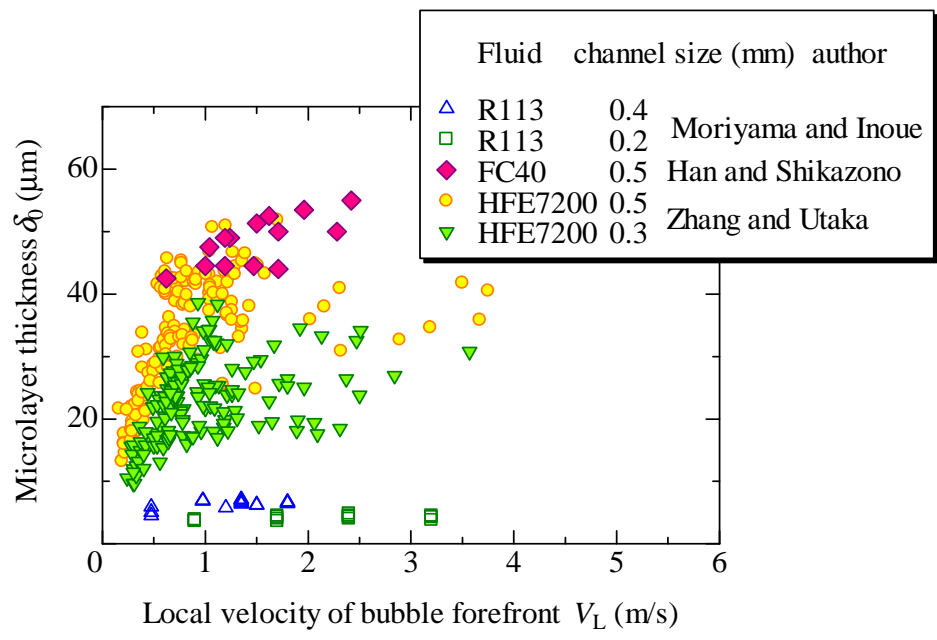

Fig. 8 Comparison of initial microlayer thickness measured by different methods.

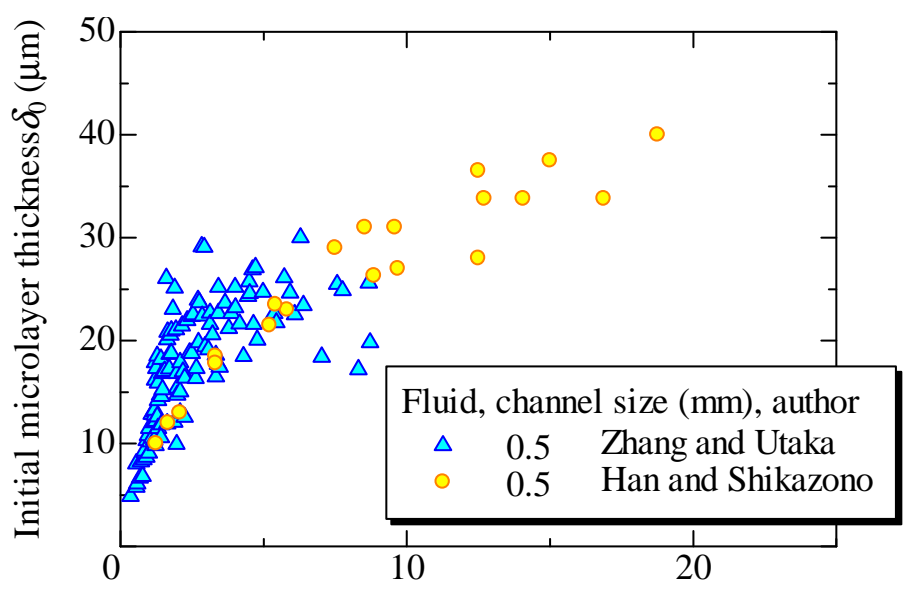

Local velocity of bubble forefront $V_{L}(\mathrm{~m} / \mathrm{s})$

Fig. 9 Comparison of microlayer thickness of water with channel size of $0.5 \mathrm{~mm}$ measured by Zhang and Utaka (2010a), and Han and Shikazono (2010a)

Table 2. Properties of the test fluids at $1 \mathrm{~atm}$ and saturation point

\begin{tabular}{|c|c|c|c|}
\hline \multicolumn{2}{|c|}{$\rho\left[\mathrm{kg} / \mathrm{m}^{3}\right]$} & $\mu[\mu \mathrm{Pa} \cdot \mathrm{s}]$ & $\sigma[\mathrm{mN} / \mathrm{m}]$ \\
\hline HFE7200 & 1307 & 330.0 & 9.4 \\
\hline R113 & 1499 & 515.7 & 14.8 \\
\hline $\mathrm{FC} 40$ & 1849 & 326.7 & 16 \\
\hline
\end{tabular}

\subsection{STEADY SLOW MOTION}

Early research on the microlayer between a bubble and capillary tube wall was related to fields such as enhanced oil recovery and monolithic catalyst reactors. Most of these involve adiabatic bubble flow with low rates and narrow channels; therefore, the microlayer thickness was considered to be determined only by the balance of surface tension and viscous forces, so that the Navier-Stokes equations could be simplified to the Stokes equation with the assumption of creeping flow.

Bretherton (1961) pioneered a theory for vanishingly small capillary numbers by assuming that the bubble acts as a tight-fitting piston with a microlayer lubricating it and moves slowly (Reynolds number, $R e \ll 1$ ). The domain is divided into a constant-microlayer-thickness region and a bubble nose region. In the constant-microlayer-thickness, the problem is solved by regular perturbation theory. In the bubble nose region, the interface is assumed to be almost hydrostatic and almost circular. The interfacial tension is important in this region. These two regions must be continuously connected with each other, so that there must be a transition region in which the shape is deformed by viscous traction so that viscous forces also become important. The method of matched asymptotic expansions is used between two sub-regions; the capillary static region where the interface is an almost spherical cap and the transition region where the lubrication approximation can be applied. The fraction of liquid deposited on the walls by a wetting bubble was found to follow the well-known law, $W=1.29(3 C a)^{2 / 3}$, where $C a$ is the capillary number. However, the agreement between theory and experiment was less than satisfactory. For $\mathrm{Ca}>10^{-4}$, the predicted law was approximately obeyed, but at slower bubble speeds, the measured microlayer thickness significantly exceeded the theoretical thickness.

Katto and Shoji (1970) considered a liquid-layer flowing radially along a solid plane, as shown in Fig. 10, where $r$ represents the radial coordinate; $y$ is the vertical coordinate; $h$ is the microlayer thickness at location $r$; and $u$ is the horizontal component of the liquid velocity at any height in the liquid layer. The volumetric flow rate of liquid through a control surface 1-2, which is a cylindrical surface, is given by $2 \pi r V$, where $V=\int_{0}^{h} u d y$. The difference between the mass of liquid flowing into and out of control volume 1-2-3-4 was considered as being balanced by the change in the height of the liquid layer. The equation of continuity was then derived as

$$
\frac{1}{r} \frac{\partial(r V)}{\partial r}=-\frac{\partial h}{\partial t}
$$

The gas that is in contact with the liquid layer was assumed to be kept at a uniform pressure, and the inertial force and gravity were neglected; these are similar assumptions to those employed by

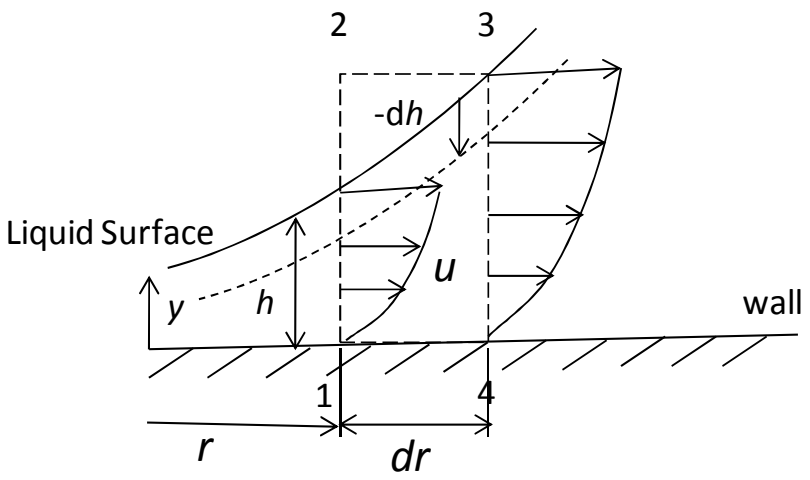

Fig.10 Radial flow of liquid along a solid plane by Katto and Shoji (1970). 
Bretherton (1961). The liquid layer is so thin, so that the pressure generated by the surface tension can be assumed to extend immediately across the liquid layer. The pressure gradient within the liquid layer produced by the change in the curvature is balanced with the viscous resistance to flow. For the equation of interface, it was assumed to descend by an infinitesimal quantity of $d h$ in an infinitesimal interval of $\mathrm{d} t$. By assuming the interface curvature $k$ to be

$$
k=\frac{d^{2} h}{d r^{2}} /\left[1+\left(\frac{d h}{d r}\right)^{2}\right]^{\frac{1}{2}} \approx \frac{d^{2} h}{d r^{2}}
$$

with the presupposition that $d h / d r \ll 1$, the microlayer formation is given in the following form

$$
\frac{d h}{d r}=\text { const } .\left(\frac{\mu U}{\sigma}\right)^{(1+\alpha) / 3} \frac{H}{r}
$$

where $\alpha$ is a numerical constant. This theoretical analysis is consistent with the correlation obtained by the experiment conducted in the same research using const. $=0.8$ and $\alpha=1$.

Reinelt and Saffman (1985) examined the steady-state shape of a finger penetrating into a viscous fluid by solving two-dimensional Stokes equations for low $R e$ flow. The equations were solved numerically by covering the domain with a composite mesh composed of a curvilinear grid on the curved interface, and a rectilinear grid parallel to the straight boundaries. The shape of the finger was altered to satisfy the normal-stress boundary condition by employing a nonlinear least squares iteration method. They considered plane and axisymmetric flow geometries, and in both cases, the results agreed well with the results of Taylor (1961) for the range of $0.01<C a<2$.

Tsai and Miksis (1994) analyzed the axisymmetric creeping flow problem by numerically solving the Stokes equations using a boundary integral method. They integrated the kinematic condition to match in time from an assumed initial bubble shape until the final steady state was attained. The results indicate that the ratio of bubble and liquid viscosities does not significantly affect the bubble shape. Both the overall length and the curvature near the front of the bubble increase with increasing $C a$, whereas the curvature at the trailing interface decreases. The bubble becomes more tapered at the front for large $\mathrm{Ca}$, which in turn increases the film thickness between the bubble and the tube wall. The computed film thickness for a viscosity ratio of 0.001 agrees well with the experimental results of Taylor (1961) (for a viscosity ratio of ca. 0.0001 ) and $C a<0.1$, but deviates slightly for $C a>0.2$. The simulation results indicate that the viscosity ratio affects the microlayer thickness and begins to appear as $\mathrm{Ca}$ increases, though the effect is weak.

For the limit of slow flow, Aussillous and Quere (2000) proposed a first-order analysis using scaling arguments. Similar to the previously described analyses, the bubble front was assumed to be spherical with radius $r$; therefore, the Laplace pressure difference across the gas-liquid interface is given by $\Delta p=2 \sigma / r$, provided the film thickness is small ( $\delta \ll r$ ). In the region of constant film thickness, the curvature in the axial direction vanishes and the Laplace pressure difference is given by $\Delta p=\sigma / r$. The balance of the viscous force and the pressure gradient in the transitional region yields

$$
\frac{\mu u}{\delta^{2}} \sim \frac{1}{\lambda} \frac{\sigma}{r}
$$

where $\lambda$ is the length of the transitional region between the spherical and flat interface, $\lambda$ is unknown, but it can be estimated by requiring that the Laplace pressure is continuous at the interface, (i.e., the curvature of the spherical part matches the curvature at the end of the transition region).

$$
-\frac{\sigma}{r}-\frac{\sigma \delta}{\lambda^{2}} \sim-\frac{2 \lambda}{r}
$$

Thus, $\lambda \sim \sqrt{\delta r}$, from which the classical scaling rule can be deduced as $\delta / r \sim \mathrm{Ca}^{2 / 3}$. This equation is valid for small capillary numbers (since $\delta \ll r$ implies that $C a \ll 1)$. As $C a$ increases, $r$ must be replaced by $(r-\delta)$ in Eqs. (6) and (7), so that the film formation scaling law becomes

$$
\frac{\delta}{r} \sim \frac{\mathrm{Ca}^{2 / 3}}{1+\mathrm{Ca}^{2 / 3}}
$$

Based on the experiment results, a good fit of Eq. (8) is found to quite precisely describe the data as

$$
\frac{\delta}{r}=1.34 \frac{\mathrm{Ca}^{2 / 3}}{1+1.34 \times 2.5 \mathrm{Ca}^{2 / 3}}
$$

where the coefficient 1.34 was derived by Bretherton (1961); the coefficient 2.5 is empirical.

In summary, several studies have used different methods to investigate steady adiabatic laminar motion of long bubbles through mini/microchannels with negligible effect of gravity. The mechanism of microlayer formation under steady, adiabatic conditions has almost been clarified. Under a slow motion limit of bubbles with negligible inertia, the microlayer thickness is determined by the balance between the viscous and capillary forces near the bubble tip; thus, the microlayer thickness (normalized by the tube radius or gap size) is only dependent on the capillary number $C a$, and approaches a finite value as $C a$ increases.

\subsection{STEADY HIGH VELOCITY FLOW}

As the steady propagation speed of a bubble increases, the inertial effects cannot be neglected in the problem. The inertial term has to be taken into account and we have to employ the steady Navier-Stokes equation instead of Stokes equation to describe the problem.

The effect of inertia was first investigated numerically by Edvinsson and Irandoust (1996) using a commercial finite element method (FEM) program (FIDAP). They found that as the flow rate and diameters are increased, the influences of inertial forces as well as gravitational forces become more pronounced. The predicted thickness of the liquid film was slightly lower than those results observed experimentally, which was attributed to a peripheral variation in the surface tension. Moreover, it was also found that as $R e$ (in the range of 20 to 2000) increases the film thickness becomes thicker, despite the effect being rather small as long as the flow is laminar.

Giavedoni and Saita (1997) used the FEM to obtain numerical results for both plane and axisymmetric cases. For the case when inertial forces are negligible $(\operatorname{Re}=0)$, the predicted microlayer thicknesses agreed well with Bretherton's asymptotic theory when $C a$ is smaller than 0.001. They also carried out computations for fixed values of $C a$, while varying $R e$ from 0 to 70 . They found that the film thickness varies non-monotonically with $R e$ for capillary values greater than 0.05 , while the film thickness was essentially independent of the inertial forces for $\mathrm{Ca}$ smaller than 0.01. This work has been extended by Heil (2001) for a two-dimensional channel case using the same numerical method as Giavedoni and Saita (1997) for a large range of $R e$. 
The results indicate a small thickening effect due to inertia for a finite Reynolds number up to 280 . The nondimensional microlayer thickness initially decreases with the increasing of $R e$ up to approximately 100 , but for larger values of $R e$ this behavior is reversed, as shown in Fig. 11.

Aussillous and Quere (2000) also investigated the inertia effect using low viscosity liquids and found that above a threshold of $\mathrm{Ca}$ the microlayer is thicker than the correlation derived from Stokes equation. They analyzed the problem by modifying the scaling laws (8) and (9). The scaling steady Navier-Stokes equation then becomes

$$
\frac{\mu u}{\delta^{2}} \sim \frac{1}{\lambda}\left(\frac{\sigma}{r-\delta}\right)-\frac{1}{\lambda} \rho u^{2}
$$

where $\rho$ is the liquid density. Similarly, matching between spherical and flat interfaces gives

$$
-\frac{\sigma}{r-\sigma}-\frac{\sigma \delta}{\lambda^{2}} \sim-\frac{2 \lambda}{r-\delta}+\rho u^{2}
$$

Finally, by eliminating $\lambda$ in Eq. (13), the following will be obtained

$$
\frac{\delta}{r} \sim \frac{\mathrm{Ca}^{2 / 3}}{1+\mathrm{Ca}^{2 / 3}-\mathrm{We}}
$$

The dimensionless number that quantifies the importance of inertia is the Weber number $W e$, defined as: $W e=\rho u^{2}(r-\delta) / \sigma$. Equation (12) agrees with the trend that the inertia thickens the film thickness; however, it seems that the coefficient and exponent require modification based on experimental or numerical results, otherwise it is only valid for $W e<1$.

Ryck (2002) presented an extension of the problem for $R e$ up to 1000 , based on a regular perturbation method. The velocity dependence of the film thickness was obtained by numerically integrating the film profile and searching for the maximum deposited film thickness that permits matching with a spherical tip. Thickening due to inertia was observed for low viscosity liquids. Figure 12 shows a plot of thenormalized thickness $E=\delta / r$ (i.e., the ratio between the film thickness $\delta$, and the tube radius $r$ ) as a function of $F=\rho \sigma r / \mu^{2}$, which depends only on the nature of the liquid and the tube radius. In particular, it can be noticed that the thinning, which may attain $20 \%$ for

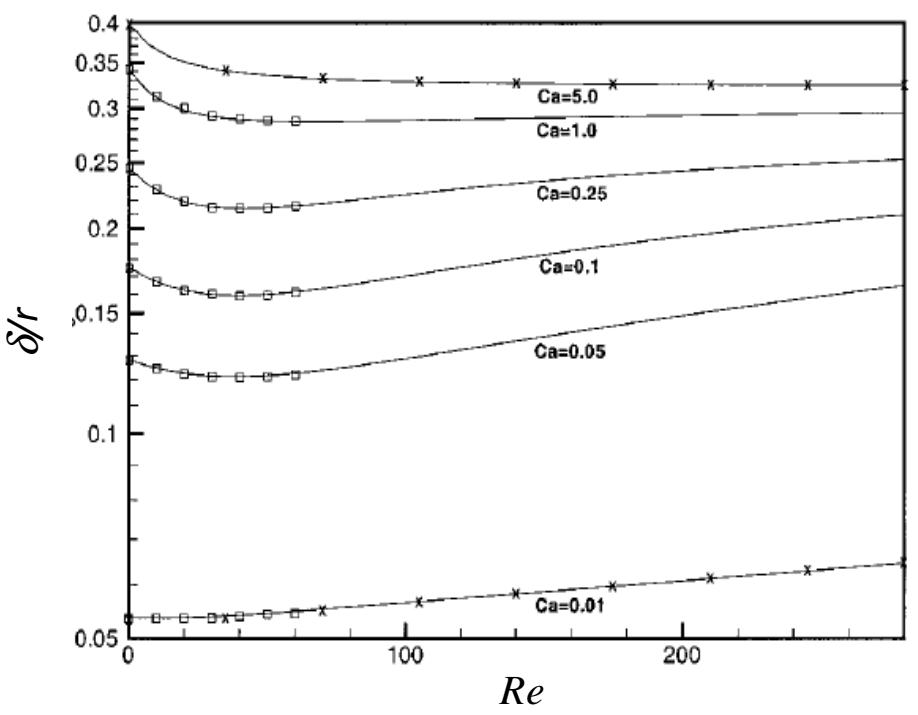

Fig. 11 Effect of the Reynolds number by Heil (2001).

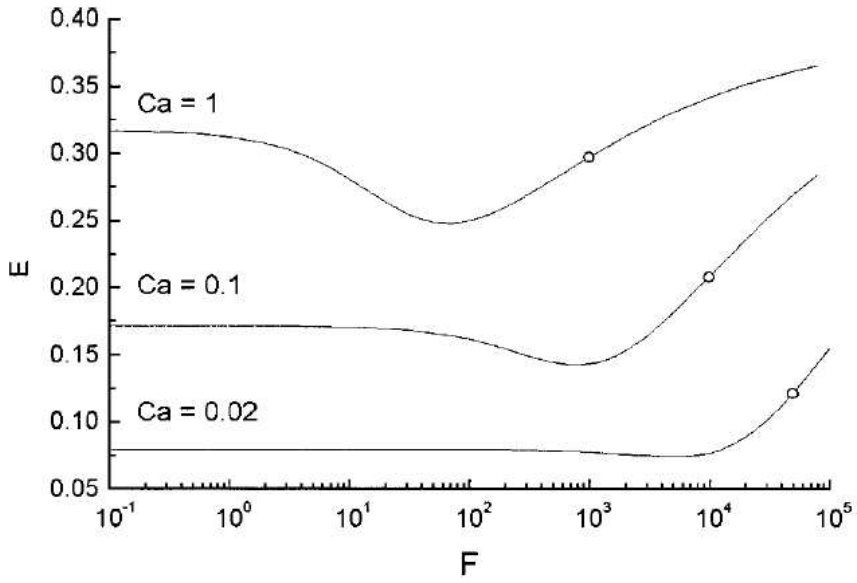

Fig. 12 Normalized thickness $\mathrm{E}=\delta / r$ vs. $\mathrm{F}=\rho \sigma r / \mu^{2}$ for three capillary numbers. The circles indicate $R e=1000$ by Ursenbacher et al. (2004).

$C a=1$, is maximum for $R e$ around 100 , and the reversed trend from thinning to thickening appears early as $\mathrm{Ca}$ increases.

Based on the scaling analysis of Eq. (12) and numerical simulation results for the effect of Re, Han and Shikazono (2009) assumed the effect of inertial force could be expressed by adding a function of $R e$ and $\mathrm{Ca}$ to the curvature as

$$
k=\frac{1+f(\operatorname{Re}, C a)}{R-\delta}
$$

Then the following relation was obtained as

$$
\frac{\delta}{r} \sim \frac{C a^{2 / 3}}{1+f(R e, C a)-g(W e)+C a^{2 / 3}}
$$

Finally, using the least linear squares method, their experimental data was correlated in the form as:

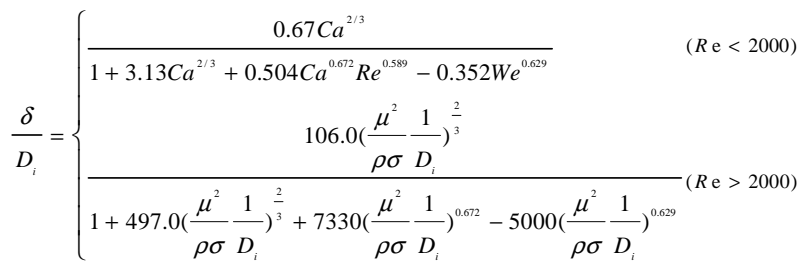

\subsection{ACCELERAED MOTION UNDER BOILING CONDITION}

There are not sufficient published studies of the accelerated motion of bubbles confined in mini/microchannels relevant to boiling when the bubble grows nonlinearly due to rapid evaporation of the microlayer (Fig. 13, by Utaka. et al., 2009). This requires using the full NavierStokes equations rather than the Stokes equations or Navier-Stokes equations that neglect local acceleration associated with unsteady flow.

In the study of Moriyama and Inoue (1996), it was observed that the microlayer thickness exhibits two different trends as the bubble forefront velocity increases as mentioned in section 2 . They considered that a transition in the controlling mechanism occurred within the range 
of their experimental conditions. Two regimes for microlayer formation in their experimental study were identified according to whether the Bond number, $B o_{a}=\frac{\rho D_{i}^{2}}{\sigma}\left(\frac{U^{2}}{2 D}\right)$ (which is calculated with approximately constant acceleration of interface movement instead of gravity) is greater than 2 as Eq. (16), where $s$ is the distance between the two parallel plates; $C a$ is defined as $C a=\frac{\mu_{L} U}{\sigma} ; t_{g}$ is bubble growth time; $\mu_{L}$ and $\rho_{L}$ are the viscosity and density of the liquid, respectively.

$$
\frac{\delta}{s}= \begin{cases}0.07 C a^{0.41} & \left(B o_{\text {a }} \leq 2\right) \\ 0.10\left[\frac{1}{s} \sqrt{\frac{\mu_{L} t_{g}}{\rho_{L}}}\right]^{0.84} & \left(B o_{\text {a }}>2\right)\end{cases}
$$

For small $B o_{a}$, the dependence of $\delta / s$ only on $C a$ suggests that the steady-flow correlation with negligible inertia should apply, while the microlayer formation is controlled by the viscous boundary layer when $B o_{a}$ is greater than 2 (see Fig. 14). As noted by Moriyama and Inoue (1996), their correlation gives values about three times smaller than the theoretical correlation (broken line) proposed by Park and Homsy (1984) for the range of conditions in their study. They suggested that this could be because the condition of their experiment lay outside of Park and Homsy's theoretical correlation and the exponent of $\mathrm{Ca}$ decreased when $\mathrm{Ca}$ increased. This work is the first to provide experimental results that bridge the two physical regions of microlayer formation, and should be considered as one of the milestones for microlayer formation mechanism research.

Han and Shikazono (2010a) assumed that under accelerated conditions bubble nose curvature is affected by the viscous boundary layer. Based on the measured results of the microlayer thickness affected by acceleration as mentioned in section 2, a modification coefficient $f=0.692 B o_{a}^{0.414}$ was obtained by least squares fitting of the data at $\mathrm{Bo}_{\mathrm{a}}>1$. Then a correlation using the Bond number, $B o_{\mathrm{a}}$, defined as the same as Eq. (16), and $\mathrm{Ca}$ for the data $\mathrm{at}_{\mathrm{a}}>1$ is proposed as

$$
\frac{\delta}{D}=\frac{0.968 \mathrm{Ca}^{2 / 3} \mathrm{Bo}_{a}^{-0.414}}{1+4.838 \mathrm{Ca}^{2 / 3} \mathrm{Bo}_{a}^{-0.414}}
$$

Their experimental data was found to be predicted accurately within the range of $15 \%$ accuracy using this correlation.

Zhang et al. (2010b) proposed a non-dimensional correlation by applying a similar method to that by Moriyama and Inoue (1996) for generally understanding the underlying mechanism of microlayer formation. Analysis of the experimental results showed that the boundaries of the two regions correspond to $W e=\rho_{L} V_{L}^{2} d_{h} / \sigma$ (based on a hydrodynamic diameter $d_{h}=2 s$ ) of approximately 110 , as shown in Fig.15, where $\delta_{V n}$ is the dimensionless viscous boundary layer thickness defined as ( $D$ is the distance from the incipient bubble site):

$$
\delta_{V n}=\frac{\delta_{V}}{2 s}=\frac{1}{2 s} \sqrt{\frac{\mu_{L} D}{\rho V_{L}}}
$$

In the region where $W e$ was smaller than 110 , the nondimensional thickness of the microlayer increases almost linearly with We. However, for the region where $W e$ was larger than 110 , the nondimensional microlayer thickness is almost independent of $W e$, where the microlayer thickness seems to obey the Prandtl law.

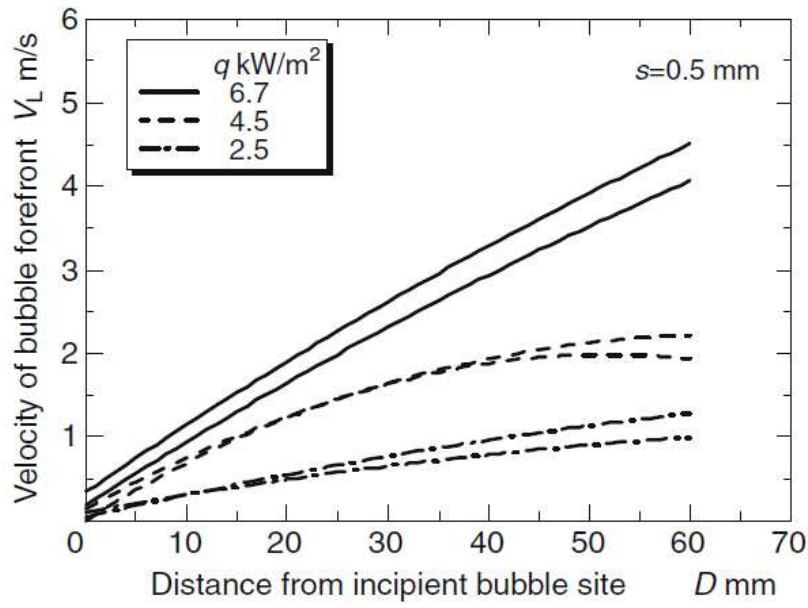

Fig.13 Increase in the bubble forefront velocity with distance from the bubble site by Utaka et al. (2009).

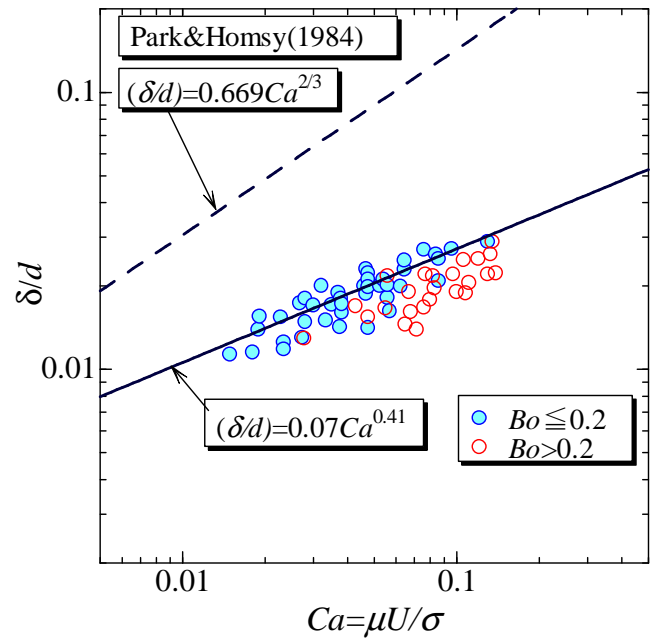

Fig.14 Correlation of the microlayer thickness with the capillary number by Moriyama and Inoue (1996).

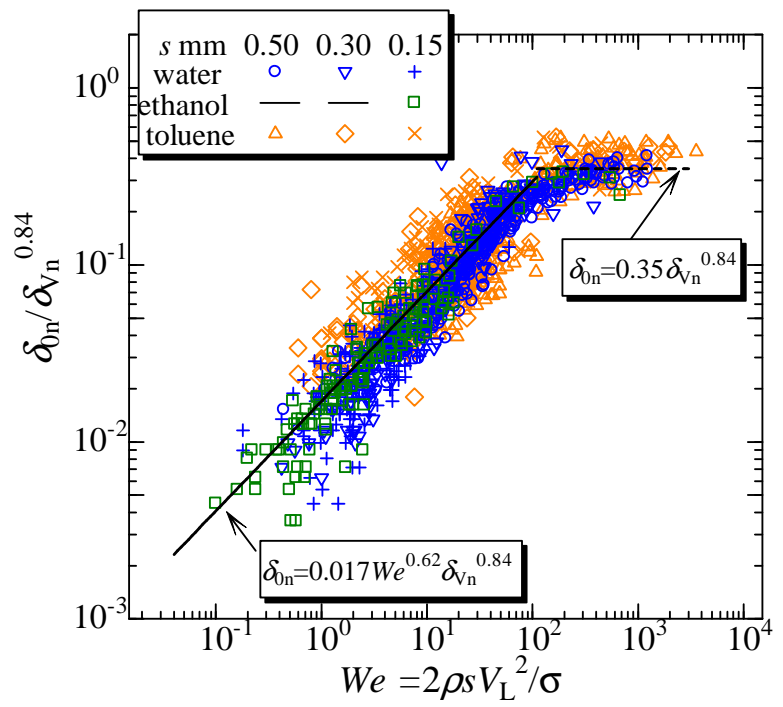

Fig.15 Nondimensional microlayer thickness versus Weber number for water, ethanol and toluene with gap sizes of $0.15,0.3$, and $0.5 \mathrm{~mm}$ by Zhang et al. (2010b). 


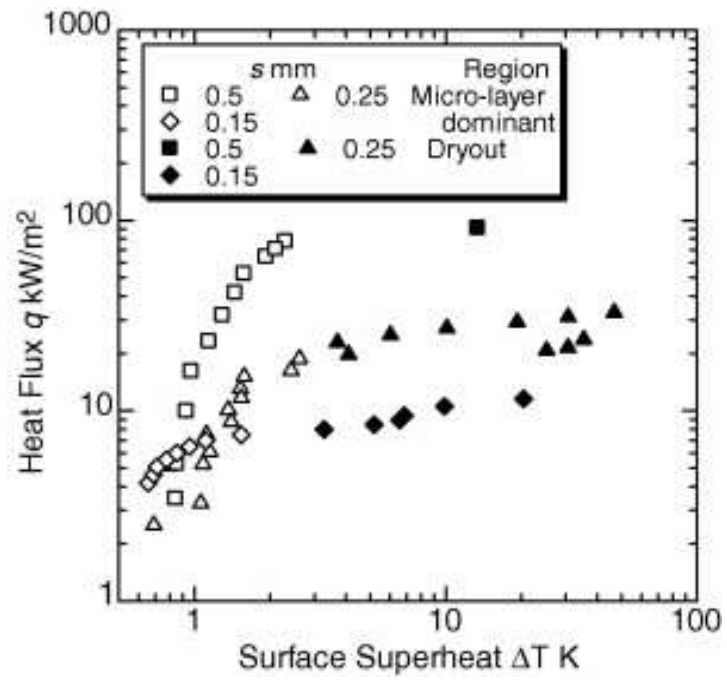

Fig.16 Effect of gap size on the boiling curves on a titanium oxidecoated surface by Tasaki and Utaka (2003).

Generally, the pattern of two regions (i.e. surface tension-viscosity control region and boundary layer control region) seems acceptable to describe the microlayer formation for a wide range of bubble growth velocity. However, the important parameter used in these correlations is that the distance of a bubble traveling from the incipient site is still difficult to predict; therefore, accurate prediction of microlayer thickness based on the basic parameter of a microchannel boiling system still requires development.

\section{EFFECT OF MICROLAYER EVAPORATION ON BOILING HEAT TRANSFER IN MICROCHANNELS}

Tasaki and Utaka (2003) measured boiling curves for water in mini/microchannels formed between two parallel quartz glass plates with a titanium oxide-coated wetting surface, as shown in Fig. 16. The curves can almost be divided into a microlayer evaporation region and dryout region. In the microlayer evaporation region, heat transfer was enhanced due to the formation and sustaining of a microlayer on the heating plate with a highly wetting surface in a smaller superheat region within $3^{\circ} \mathrm{C}$. The microlayer evaporation region occupied approximately 70 to $80 \%$ of the maximum heat flux, which indicated that microlayer

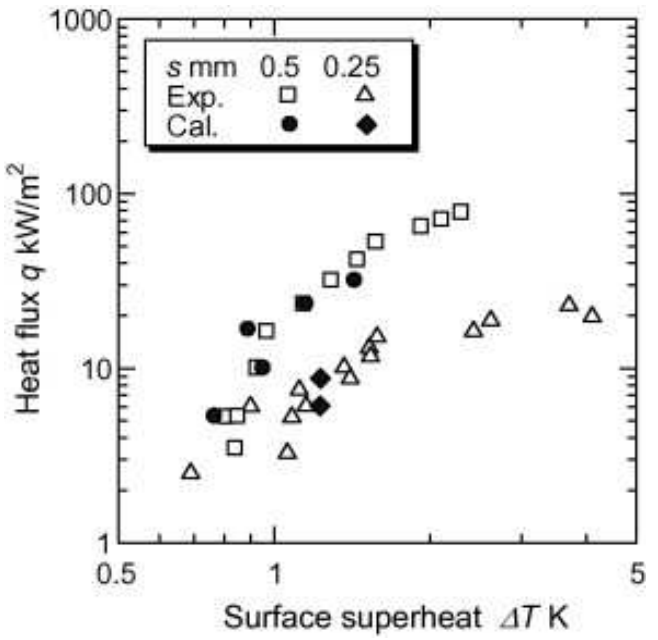

Fig.17 Comparison of experimental and calculated boiling curves by Utaka et al. (2009).

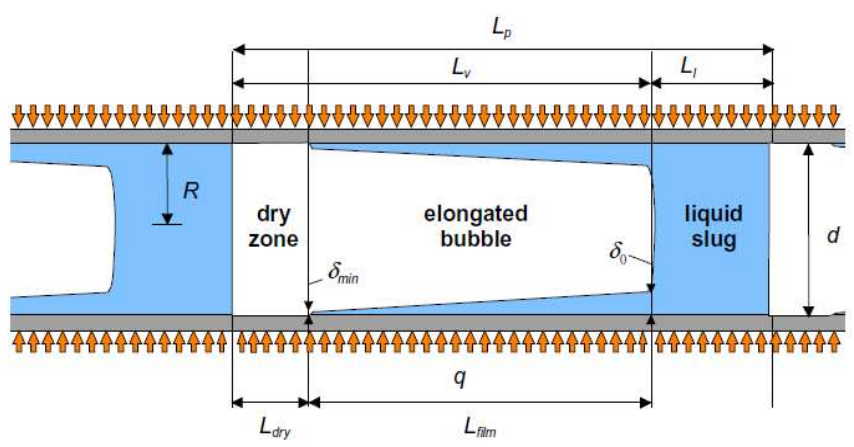

(a) Three-zone heat transfer model for the elongated bubble flow regime in a microchannel

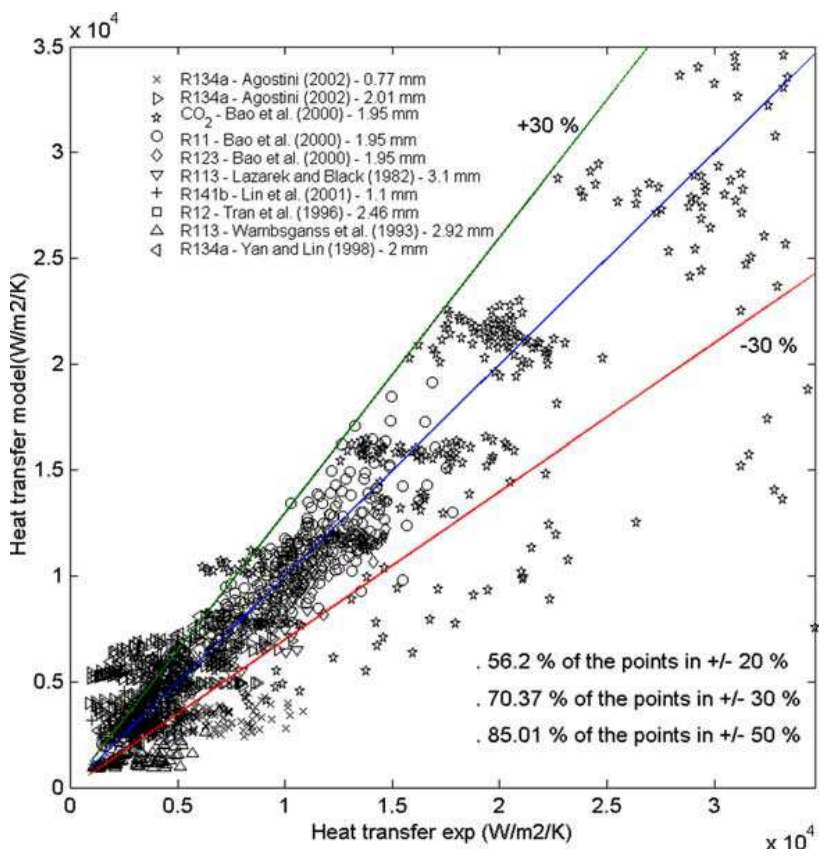

(b) Comparison of experimental heat transfer and the corresponding values given by the model

Fig.18 Schematic illustration of the three-zone heat transfer model and results for elongated bubble flow in a microchannel by Thome et al. (2004).

evaporation was the dominant heat transfer mode in microchannels.

As a continuation of the study by Tasaki and Utaka (2003), Utaka et al. (2009) divided the bubble generation cycle into microlayer and liquid saturation periods. The changes in microlayer thickness and the transition of the degree of superheat on the heat transfer surface in the microlayer period, and the temperature changes of bulk liquid in the liquid saturation period, were calculated at different heat flux for gap sizes of 0.5 and $0.25 \mathrm{~mm}$ with the assumption of one-dimensional transient heat conduction in the liquid. The calculated values agreed well with the experimental results (see Fig. 17), which confirmed that the heat transfer is enhanced due to microlayer evaporation.

Thome et al. (2004) analyzed the heat transfer coefficient in each zone (liquid slug, elongated bubble and vapor slug) of the proposed three-zone flow boiling model as shown in Fig. 18(a). Using the measurement results for microlayer thickness from Moriyama and Inoue (1996), they calculated the local heat transfer coefficient in the evaporating microlayer by assuming that the microlayer is stagnant and the heat transfer coefficient is controlled by one-dimensional conduction across the microlayer. For liquid and vapor slugs, heat transfer coefficients were calculated from their respective local Nusselt 


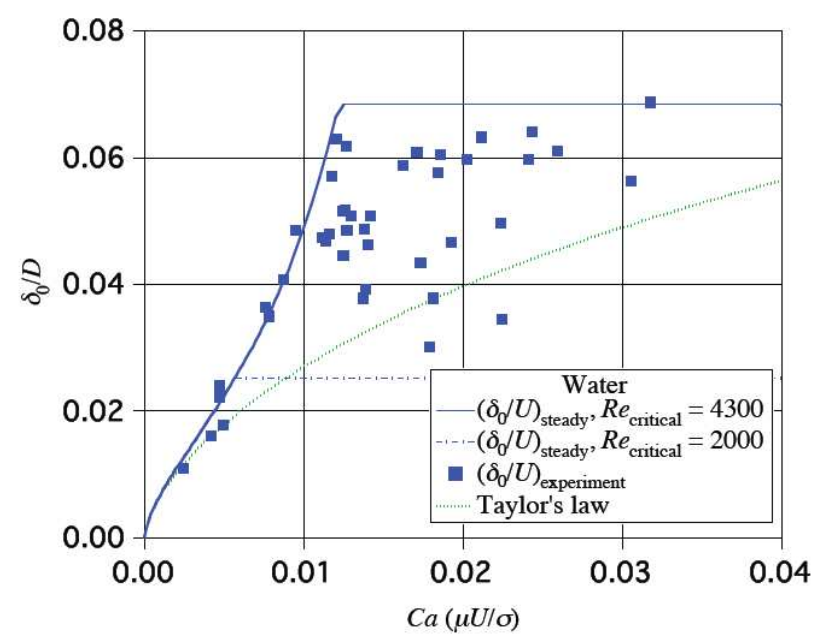

Fig.19 Dimensionless liquid film thickness versus capillary number for an experiment using water by Han et al. (2010b).

numbers and applied to the respective lengths of the liquid slug and dry wall zones. The calculation results given by the model were compared with the database, as shown in Fig. 18(b). By asymptotic method, a continuous expression of the mean heat transfer coefficient of liquid slug or dry wall zone as a function of $R e$ was proposed for both laminar developing flow and turbulent developing flow with a critical $R e$ of 2300. As reported by Kurokawa and Moriyama (1986), even a small acceleration significantly increases the critical $R e$. Under boiling condition, acceleration and deceleration (because of the expansion of the preceding bubble) occur; therefore, a higher critical $R e$ should be used during boiling in mini/microchannels. Han et al. (2010b) found that under the boiling condition, the microlayer thickness of water is thicker than the prediction of Eq. (15) (which is obtained at steady flow condition) at $R e_{\text {critical }}=2000$, but follows the prediction when $R e_{\text {critical }}=4300$ is used (see Fig. 19). Similarly, by assuming onedimensional transient heat conduction, they calculated heat transfer coefficients from measured microlayer thickness for a circular tube. The results show good accordance with the heat transfer coefficients obtained directly from wall temperature measurements at small vapor qualities.

The heat transfer coefficient could generally be predicted well by the assumption of one-dimensional transient heat conduction if the initial microlayer thickness is given exactly. Therefore, accurate models or methods including the transient nature of flow to predict the thickness of microlayers and the other effects on the boiling heat transfer coefficients in microchannels still require further investigation.

\section{SUMMARY AND CONCLUSIONS}

Experiments, numerical simulations, and theoretical analyses of microlayers formed during boiling in mini/microchannels have been reviewed.

Several techniques have been attempted to measure the microlayer thickness in mini/microchannels under both adiabatic and boiling conditions with accurate measurement results obtained in their respective experimental systems. A uniform standard of appraisal for measurement results obtained by different methods still requires development in the future.

Bubble motion in mini/microchannels for laminar, steady, and adiabatic conditions has been almost completely clarified, both theoretically and experimentally, and methods for the prediction of microlayer thickness have also been successfully developed for these conditions. However, there is only a small amount of publications available on the microlayer formation mechanism during boiling in mini/microchannels. The effects of inertia and acceleration must be taken into account for the potentially highly transient vapor bubble growth in mini/microchannels. Generally, the pattern of two regions (i.e. surface tension-viscosity control region and boundary layer control region) seems acceptable to describe the microlayer formation for a wide range of bubble growth velocity.

\section{REFERENCES}

Ameur, M.A., Stutz, B. and Lallemand, M., 2007, "Unsteady natural convective boiling in a narrow vertical channel", Heat Transfer Eng., 28, 870-876. http://dx.doi.org/doi:10.1080/01457630701378317

Aussillous, P. and Quere, D., 2000, "Quick deposition of a fluid on the wall of a tube", Phys. Fluids, 12, 2367-2371.

http://dx.doi.org/doi:10.1063/1.1289396

Bretherton, F.P., 1961, "The motion of long bubbles in tubes", J. Fluid Mech., 10, 166-188.

http://dx.doi.org/doi:10.1017/S0022112061000160

Carvalho, R.D.M., Ventruini, O.J., Neves, J.F. and Franca, F.A., 2008, "Axial bubbly flow topology: A comparative study using the ultrasonic technique and high-speed filming", in Proceedings of the $5^{\text {th }}$ International Conference on Transport Phenomena in Multiphase Systems, Bialystok, Poland.

Cheng, P., Wu, H.Y. and Hong, F.J., 2007, "Phase-Change Heat Transfer in Microsystems", J. Heat Transfer, 129 101-107. http://dx.doi.org/doi:10.1115/1.2410008

Chen, Y., Kulenovic, R. and Mertz, R., 2009, "Numerical study on the formation of Taylor bubbles in capillary tubes", Int. J. Therm. Sci., 48, 234-242.

http://dx.doi.org/doi:10.1016/j.ijthermalsci.2008.01.004

Edvinsson, R.K. and Irandoust, S., 1996, "Finite-element analysis of Taylor flow", AIChE J., 42(7), 1815-1823.

http://dx.doi.org/doi:10.1002/aic.690420703

Giavedoni, M.D. and Saita, F.A., 1997, "The axisymmetric and plane cases of gas phase steadily displacing a Newtonian liquid-A simulation solution of governing equation", Phys. Fluids, 9, 2420.

http://dx.doi.org/doi:10.1063/1.869360

Han, Y. and Shikazono, N., 2009, "Measurement of the liquid film thickness in micro tube slug flow", Int. J. Heat Fluid Flow, 30(5), 842853.

http://dx.doi.org/doi:10.1016/j.ijheatfluidflow.2009.02.019

Han, Y. and Shikazono, N., 2010a, "The effect of bubble acceleration on the liquid film thickness in micro tubes", Int. J. Heat Fluid Flow, 31, 630-639.

http://dx.doi.org/doi:10.1016/j.ijheatfluidflow.2010.02.002

Han, Y., Shikazono, N. and Kasagi, N., 2010b, "The effect of liquid film evaporation on flow boiling heat transfer in a micro tube", in Proceedings of the $14^{\text {th }}$ International heat transfer conference.

Heil, M., 2001, "Finite Reynolds number effects in the Bretherton problem", Phys. Fluids, 13(9), 2517-2521.

http://dx.doi.org/doi:10.1063/1.1389861 
Jacobi, A.M. and Thome, J.R., 2002, "Heat transfer model for evaporation of elongated bubble flows in microchannels", J. Heat Transfer, 124, 1131-1136.

http://dx.doi.org/doi:10.1115/1.1517274

Jong, P.D. and Gabriel, K.S., 2003, "A preliminary study of two-phase annular flow at microgravity: experimental data of film thickness", Int. J. Multiphase Flow, 29, 1203-1220.

http://dx.doi.org/doi:10.1016/S0301-9322(03)00085-5

Kang, H.C. and Kim, M.H., 1992, "The development of a flush-wire probe and calibration method for measuring liquid film thickness", Int. J. Multiphase Flow, 18, 423-437.

http://dx.doi.org/doi:10.1016/0301-9322(92)90026-D

Kandlikar, S.G., 2002, "Fundamental issues related to flow boiling in minichannels and microchannels", Exp. Therm Fluid Sci., 26, 389-407. http://dx.doi.org/10.1016/0301-9322(92)90026-D

Kandlikar, S.G., 2004, "Heat transfer mechanisms during flow boiling in microchannels", J. Heat Transfer, 126, 8-16

http://dx.doi.org/doi:10.1016/S0894-1777(02)00150-4

Katto, Y., and Shoji, M., 1970, "Principal mechanism of micro-liquidlayer formation on a solid surface with a growing bubble in nucleate boiling”, Int. J. Heat Mass Transfer, 13, 1299. http://dx.doi.org/doi:10.1016/0017-9310(70)90071-2

Kurokawa, J. and Moriyama, M., 1986, "Accelerated and decelerated flows in a circular pipe", Bulletin of JSME, 29 (249), 758-765.

Kumar, R., Gottmann, M. and Sridhar, K.R., 2002, "Film thickness and wave velocity measurements in a vertical duct", J. Fluids Eng., 124, 634-642.

http://dx.doi.org/doi:10.1115/1.1493808

Liu, S., Li J. and Chen, Q., 2007, "Visualization of flow pattern in thermosyphon by ECT", Flow Meas. Instrum., 18, 216-222. http://dx.doi.org/doi:10.1016/j.flowmeasinst.2007.06.012

Lu, Q., Suryanarayana, N.V. and Christodoulu, C., 1993, "Film thickness measurement with an ultrasonic transducer", Ex. Therm Fluid Sci. 7, 354-361.

http://dx.doi.org/doi:10.1016/0894-1777(93)90058-Q

Miler, L.J., Ahmed, G.R., Touzelbaev, M.N., David, M.P., Steinbrenner, J.E. and Goodson K.E., 2010, "Reduced-Order Fluidic Model For Flow Instabilities in Two-Phase Microfluidic Heat Exchangers", in Proceedings of the $8^{\text {th }}$ international Conference on Nanochannels, Microchannels, and Minichannels, Montreal, Canada.

Moriyama, K., and Inoue, A., 1996, "Thickness of the liquid film formed by a growing bubble in a narrow gap between two horizontal plates", $J$. Heat Transfer, 118, 132-139. http://dx.doi.org/doi:10.1115/1.2824026

Ong, C.L. and Thome, J.R., 2011, "Macro-to-microchannel transition in two-phase flow: Part 1-Two-phase flow patterns and film thickness measurements", Exp. Therm Fluid Sci., 35 37-47.

http://dx.doi.org/doi:10.1016/j.expthermflusci.2010.08.004

Park, C.W. and Homsy,G.M.,1984, "Two-Phase Displacement in HeleShaw Cells:Theory",J.Fluid Mech.,139,291 http://dx.doi.org/doi:10.1017/S0022112084000367
Qu, W., and Mudawar, I., 2003, "Flow Boiling Heat Transfer in TwoPhase Microchannel Heat Sinks-I. Experimental Investigation and Assessment of Correlation Methods", Int. J. Heat Mass Transfer, 46, 2755-2771.

http://dx.doi.org/doi:10.1016/S0017-9310(03)00041-3

Reinelt, D.A. and Saffman, P.G., 1985, "The penetration of a finger into a viscous fluid in a channel and tube", SIAM J. Sci. Stat. Comput., 6, 542. http://dx.doi.org/doi:10.1137/0906038

Roy, R.P., Ku J., Kaufman, I. and Shukla, J., 1986, "Microwave method for measurement of liquid film thickness in gas-liquid flow", Rev. Sci. Instrum.,57, 952-956. http://dx.doi.org/doi:10.1063/1.1138841

Ryck, A., 2002, "The effect of weak inertia on the emptying of a tube", Phys. Fluids, 14(7), 2102-2108.

http://dx.doi.org/doi:10.1063/1.1480267

Seleghim, P. J. and Hervieu, E., 1998, "Direct imaging of two-phase flows by electrical impedance measurements", Meas. Sci. Technol., 9, 1492-1500.

http://dx.doi.org/doi:10.1088/0957-0233/9/9/018

Shedd, T.A. and Newell, T.A., 2004, "Characteristics of the liquid film and pressure drop in horizontal, Annular, Two-phase flow through round, square and triangular tubes", J. Fluids Eng., 126, 807-817. http://dx.doi.org/doi:10.1115/1.1792261

Stephan, P., 2002, "Microscale evaporative heat transfer: modeling and experimental validation", in Proceedings of $12^{\text {th }}$ International Heat Transfer Conference.

Tasaki, Y. and Utaka, Y., 2003, "Effect of wettability on boiling heat transfer characteristics in micro-channel vapor generator", Int. J. Transp. Phenom., 5(4), 295-302.

Taylor, G.I., 1961, "Deposition of a viscous fluid on the wall of a tube", J. Fluid Mech., 10, 1161-1165. http://dx.doi.org/doi:10.1017/S0022112061000159

Thwaites, G.R., Kulov, N.N. and Nedderman, R.M., 1976, "Liquid film properties in two-phase annular flow", Chem. Eng. Sci., 31, 466-481. http://dx.doi.org/doi:10.1016/0009-2509(76)80033-4

Thorncroft, G.E. and Klausner, J.F., 1997, "A Capacitance sensor for two-phase liquid film thickness measurements in a square duct", $J$. Fluids Eng.,119, 164-169.

http://dx.doi.org/doi:10.1115/1.2819103

Thome, J.R., Dupont, V. and Jacobi, A.M., 2004, "Heat Transfer model for evaporation in microchannels. Part I: presentation of the model". Int. J. Heat Mass Transfer, 47, 3375-3385.

http://dx.doi.org/doi:10.1016/j.ijheatmasstransfer.2004.01.006

Tibirica, C. B., Nascimento, F. J. and Ribatski, G., 2010, "Film thickness measurement techniques applied to micro-scale two-phase flow systems", Exp. Therm Fluid Sci., 34 463-473.

http://dx.doi.org/doi:10.1016/j.expthermflusci.2009.03.009

Tsai, T.M. and Miksis, M.J., 1994, "Dynamics of a drop in constricted capillary tube", J. Fluid Mech., 274, 197. http://dx.doi.org/doi:10.1017/S0022112094002090

Ursenbacher, T., Wojtan, L. and Thome, J.R., 2004, "Interfacial measurements in stratified type of flow. Part I: New optical 
measurement technique and dry angle measurements", Int. J. Multiphase Flow, 30,107-124.

http://dx.doi.org/doi:10.1016/j.ijmultiphaseflow.2003.11.010

Utaka, Y., Tasaki, Y. and Okuda, S., 2006, "Behavior of Micro-layer in Micro-channel Boiling System Applying Laser Extinction Method", Heat Transfer - Asian Research, 35(1), 35-46. http://dx.doi.org/doi:10.1002/htj.20096

Utaka, Y., Okuda, S. and Tasaki, Y., 2009, "Configuration of the microlayer and characteristics of heat transfer in a narrow gap mini/microchannel boiling system", Int. J. Heat and Mass Transfer, 52, 2205-2214. http://dx.doi.org/doi:10.1016/j.ijheatmasstransfer.2008.11.020

Wang, H., Garimelle, S. V. and Murthy, J. Y., 2007,"Characteristics of an evaporating thin film in a microchannel", Int. J. Heat Mass Transfer, 50, 3933-3942.

http://dx.doi.org/doi:10.1016/j.ijheatmasstransfer.2007.01.052
Wayner, P.C. Jr., "Intermolecular forces in phase-change heat transfer: Kern award review", AIChE J. 45(10), 2005-2068.

Yu, S.C.M., Tso, C.P. and Liew, R., 1996, "Analysis of thin film thickness determination in two-phase flow using a multifiber optical sensor", Appl. Math. Modell., 20, 540-548.

http://dx.doi.org/doi:10.1016/0307-904X(95)00173-H

Zhang, Y.H. and Utaka, Y., 2010a "Formation Mechanism and Characteristics of liquid Microlayer in mini-gap boiling system", in Proceedings of the $8^{\text {th }}$ international Conference on Nanochannels, Microchannels, and Minichannels, Montreal, Canada.

Zhang, Y.H., Utaka, Y. and Kashiwabara, Y., 2010b, "Formation Mechanism and Characteristics of a Liquid Microlayer in Microchannel Boiling System", J. Heat Transfer, 132(12), 122403-1--122403-7. http://dx.doi.org/doi:10.1115/1.4002365 